\title{
Time domain reflectometry for improved Surface Acoustic Wave magnetic field sensor sensitivity
}

\author{
Aurélien Mazzamurro, Abdelkrim Talbi, Yannick Dusch, Cécile Ghouila-Houri, Philippe Pernod, \\ Olivier Bou Matar, Nicolas Tiercelin
}

Univ. Lille, CNRS, Centrale Lille, ISEN, Univ. Valenciennes, UMR 8520 IEMN, LIA LICS/LEMAC, F-59000 Lille, France

\begin{abstract}
This paper shows the use of time domain reflectometry (TDR) applied to Surface Acoustic Waves (SAW) based magnetic field sensors to improve sensitivity of these devices. The basic operating principle of such sensors is the use of a magnetostrictive material along the SAW path to induce a velocity shift when biased with a magnetic field. This velocity shift is related to the interaction of the SAW with the magnetostrictive material through $\Delta \mathrm{E}$-effect. By looking at the multiple echoes occurring after the main acoustic signal (direct transit) in a delay line configuration, it is possible to extend the interaction path, resulting in an increase of the phase shift as the acoustic wave is travelling back and forth through the magnetostrictive material. The velocity shift shape obtained under a bias magnetic field is well explained using an equivalent piezomagnetic model developed in a previous work to assess elastic stiffness constant dependency of the magnetostrictive thin film with the magnetic field. It is shown that it can improve the sensitivity of the magnetic sensor by one order of magnitude.
\end{abstract}

\section{INTRODUCTION}

Recent progress in the elaboration of new magneto-elastic materials such as multilayered piezomagnetic composites make them widely used in the development of sensors, actuators, but also solid state memories due to their large magnetostrictive coefficient, compared to single phase materials ([1], [2], [3]). More specifically, the combination of SAW and magnetostrictive material leads to the development of innovative magnetic field sensors that could be integrated in harsh environment. In such sensors, the velocity of the SAW follows the magnetoelastically-induced changes of the elastic modulus according to the bias magnetic field through the $\Delta \mathrm{E} / \mathrm{G}$-effect. This velocity shift is resulting from the coupling between the magnetization state of the magnetostrictive material and its elasticity. An equivalent piezomagnetic model was developed in a previous work ([4], [5]) to assess this magnetoelastic coupling and is briefly reported in II-A. In Section II-B is reported the manufactured SAW delay line and its electrical response. Section III shows the magnetic response of the multiple echoes received in time domain reflectometry.

\section{DESCRIPTION}

\section{A. Equivalent Piezomagnetic model}

An equivalent piezomagnetic model was developed in a previous work to study the magnetoelastic coupling in piezoelectro-magnetic composites (for further details, see [4], [5]). As a recall, the piezomagnetic equations are obtained by considering a magnetoelastic wave in a ferromagnetic thin film deposited on a piezoelectric substrate, and linearizing the coupled equations for the mechanical and magnetic systems (Newton's equation of motion and Landau-Lifshitz equation) around a ground state position of the magnetization (depending on the direction and magnitude of the bias field). The piezomagnetic equations are given by

$$
\begin{aligned}
\rho \frac{\partial^{2} u_{i}}{\partial t^{2}} & =\frac{\partial \sigma_{i j}}{\partial x_{j}}, \\
\frac{\partial b_{i}}{\partial x_{i}} & =\frac{\partial\left(\mu_{0}\left(h_{i}+m_{i}\right)\right)}{\partial x_{i}},
\end{aligned}
$$

where $\rho$ is the density of the ferromagnetic thin film, $m$, the magnetization, $h$, the magnetostatic field, $u_{i}$ is the $i^{\text {th }}$ component of the particle displacement, $x_{i}$ denotes the Eulerian coordinates and

$$
\begin{aligned}
\sigma_{i j} & =\left(C_{i j k l}+\Delta C_{i j k l}\right) \frac{\partial u_{k}}{\partial x_{l}}-q_{l i j} h_{l} \\
b_{i} & =q_{i k l} \frac{\partial u_{k}}{\partial x_{l}}+\mu_{i l} h_{l}
\end{aligned}
$$

with $C_{i j k l}$ the elastic stiffness constants and where the effective magnetic permeability $\mu_{i j}$ and elastic stiffness constants corrections $\Delta C_{i j k l}$ are given by

$$
\begin{aligned}
\mu_{i l} & =\mu_{0}\left(\delta_{i l}+\chi_{i l}\right) \\
\Delta C_{i j k l} & =b_{i j m n}\left(M_{n}^{0} q_{m k l}+M_{m}^{0} q_{n k l}\right)
\end{aligned}
$$

with $b_{i j k l}$, the magnetoelastic constants $\left(b_{1111}=b^{\gamma, 2}\right)$. The expressions of the piezomagnetic constants $q_{i j k}$ and the magnetic susceptibility $\chi_{i l}$ can be found in [6].

Therefore, the elastic stiffness constant of the magnetostrictive thin film are modified by a bias magnetic field by the quantity $\Delta C_{i j k l}$, leading to SAW propagation velocity shift due to magnetoelastic coupling. This shift is dependent of the wave polarization through the elastic stiffness constant involved in the stress/strain induced in the magnetostrictive thin film. In the following, the magnetoelastic coupling was studied on lithium tantalate $\mathrm{Y}^{\circ} 6^{\circ}$ substrate cut coated with a $\mathrm{TbCo}_{2} / \mathrm{FeCo}$ multilayered thin film. This piezoelectric substrate was chosen for its quasi-shear wave inducing mainly transverse displacement field, thus involving mainly $c_{66}$ elastic stiffness constant in wave motion equation. The dependency of the $c_{66}$ elastic stiffness constant with the magnetic field is given by (6) and reported Fig. 1. 


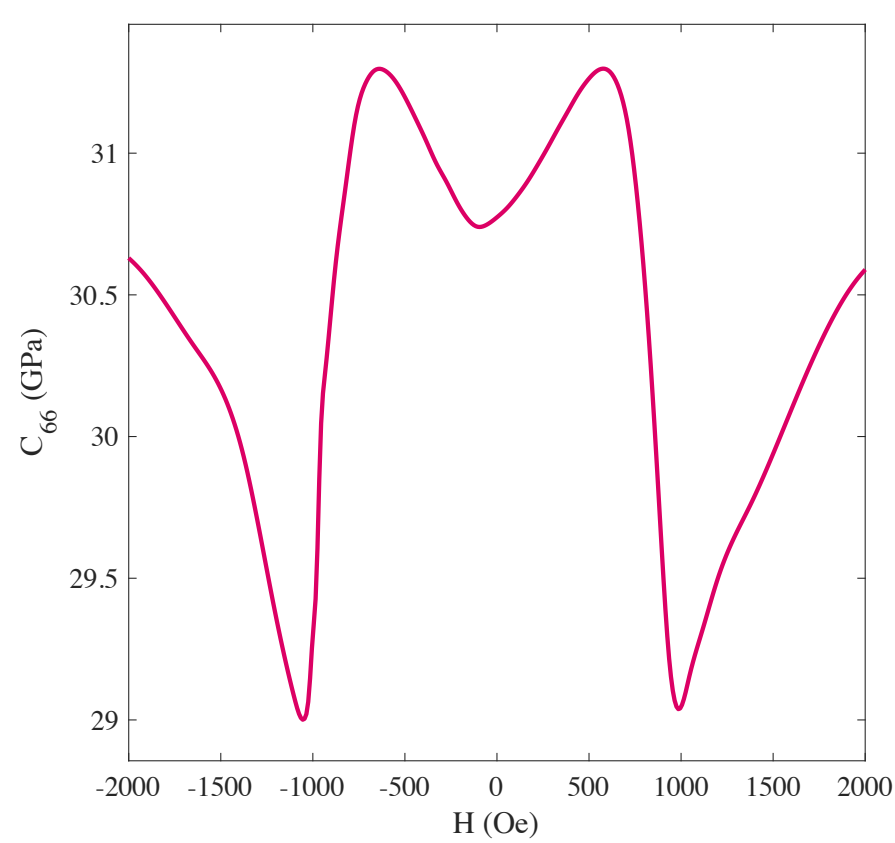

Fig. 1. Magnetic field dependency of the $C_{66}$ elastic stiffness constant obtained with the equivalent piezomagnetic model.

\section{B. Manufactured SAW device}

As mentioned above, the magnetoelastic coupling was studied in a SAW delay line magnetic field sensor that uses quasi-shear acoustic waves manufactured on lithium tantalate $\mathrm{Y} 36^{\circ}$ cut with a uniaxial multilayered $25 \times\left[\mathrm{TbCo}_{2(3.7 n m)} / \mathrm{FeCo}_{(4 n m)}\right]$ nanostructured thin film as sensitive layer, operated at $254 \mathrm{MHz}$ (see Fig. 2). In a delay line configuration, the main acoustic signal received at the output transducer is due to the direct transit of the acoustic wave launched from the input transducer. As already described, the acoustic wave is reflected back and forth between the two interdigital transducers (single electrode IDT) due to Bragg reflection leading to the well-known triple transit echo (TTE). The forthcoming echoes (fourth, fifth, sixth) contain relevant information on the environment encountered by the SAW along its propagation (transit) between the IDTs. As a general rule with SAW sensors, the longer is the interaction with the sensing layer, the better. The downside is, of course, the increase in sensor time response. Therefore, each of the echoes is showing a cumulative $\Delta \mathrm{E}$-effect compared to the previous one leading to an increase in sensitivity. Carrying measurements in time domain allows us to discriminate SAW echoes in transmission $\left(S_{21}\right)$ or reflection $\left(S_{11}\right)$.

Measurements were performed on a SAW delay line in time domain through $S_{21}$ parameter. The signal received on the output transducer through time is reported in Fig. 3. It clearly shows the different echoes corresponding to the acoustic waves being reflected between the two periodic gratings.

On this time domain response, the first peak is due to the electromagnetic pulse generated on the input transducer reaching the output transducer through the air. It is also

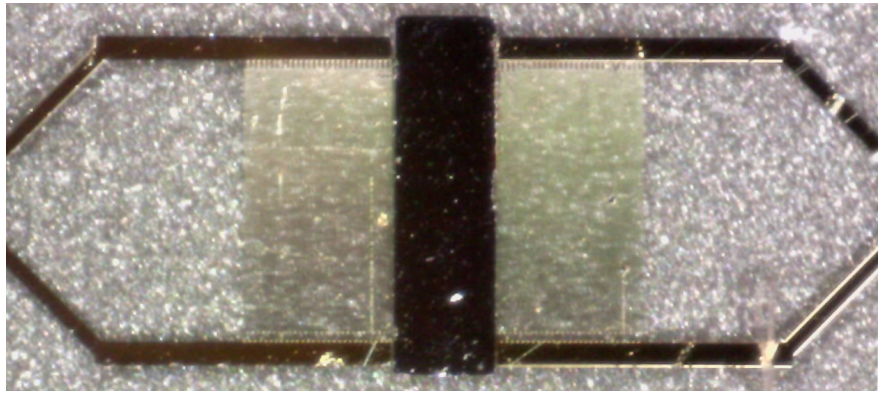

Fig. 2. Manufactured SAW delay line with the IDTs and the multilayered nanostructured magnetostrictive thin film in-between.

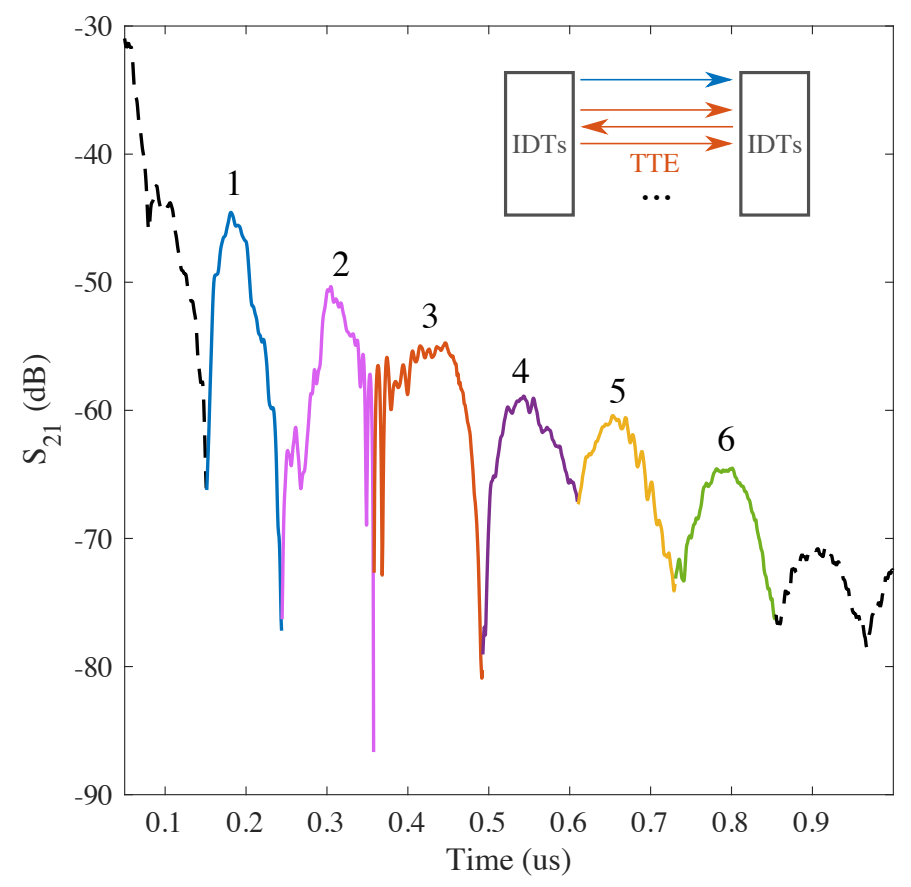

Fig. 3. Time transit delay measured on $\mathrm{S}_{2} 1$ parameter.

noticeable that echoes with an even number corresponds to the radiated response of the acoustic wave going back to the input through electromagnetic coupling. The echoes with an odd number corresponds to the acoustic wave reaching the output transducer after multiple reflections (see inset Fig. 3). The acoustic signal corresponding to a particular echo is isolated through time gating and measurements of the resulting transmission (amplitude and phase) is carried out with respect to a bias magnetic field. The results are reported in the following section.

\section{RESULTS}

In order to assess the magnetoelastic coupling effect ( $\Delta \mathrm{E}$ effect) on the SAW velocity when the magnetic sensor is biased with a varying magnetic field, the $S_{21}$ parameter was measured for the different echoes. The phase shift induced on SAW propagation is reported in Fig. 4. The relative phase shift obtained for the direct transit is around $0.05 \%$. The effect building up for the following echoes, the relative phase 


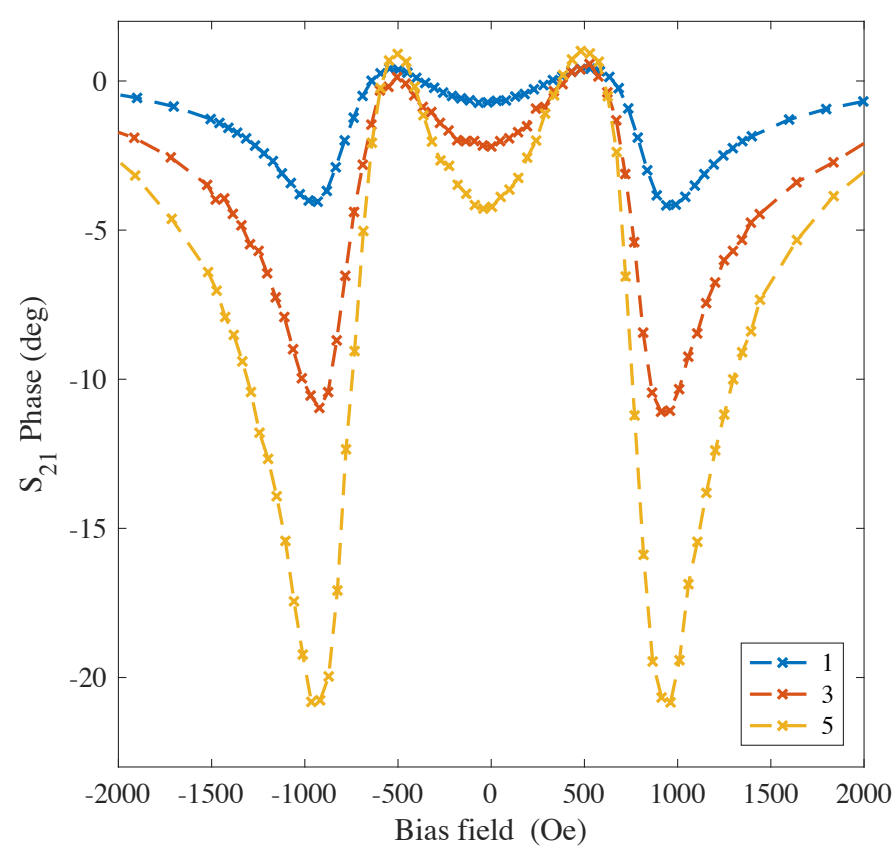

Fig. 4. $\Delta$ E-effect induced velocity shift for different transit time as reported in 3 .

shift reaches $0.25 \%$ after three travels (back and forth, or triple transit echo) through the magnetostrictive sensing layer and even $0.7 \%$ after five. Therefore, the sensitivity to the magnetic field was enhanced by a factor of ten, by simply looking at acoustic wave signals carrying the record of longer magnetoelastic interaction with the magnetostrictive sensing layer.

The theoretical predictions obtained with the described effective piezomagnetic material model, in conjunction with a numerical Legendre/Laguerre polynomial expansion method to compute propagation constants and mode shapes of elastic waves in layered piezoelectric- piezomagnetic composites deposited on a substrate [4] are reported in Fig. 5 for the considered magnetostrictive thin film. The shape of the velocity shift obtained with the equivalent piezomagnetic model is coherent with the $c_{66}$ elastic stiffness constant behaviour towards a bias magnetic field. Indeed, the bulk shear velocity in the magnetostrictive thin film (density, $\rho$ ) can be expressed as $\sqrt{c_{66} / \rho}$ for a surface acoustic wave with a shear polarization. Therefore, the evolution of the wave propagation velocity follows the variation of the $c_{66}$ elastic stiffness constant with respect to a bias magnetic field.

\section{CONCLUSION}

As a conclusion, an unusual method was proposed to enhance SAW based magnetic field sensor sensitivity. Time Domain Reflectometry allows the measure of the multiple echoes occurring in a SAW delay line between the IDTs, each of these resulting from a longer interaction path with the magnetostrictive thin film. Therefore, the overall phase shift of this kind of device when biased with a magnetic field is building up as the number of echoes increases, resulting

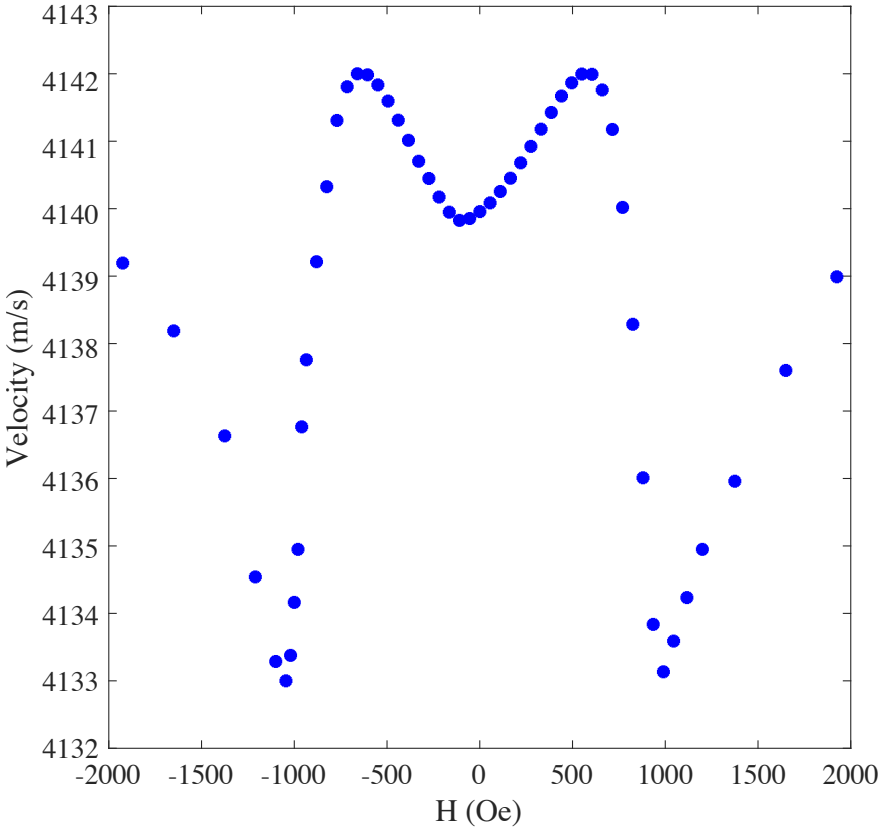

Fig. 5. Velocity shift obtained with piezomagnetic equivalent model for lithium tantalate $\mathrm{Y} 36^{\circ}$ cut.

in an increase of the phase shift for the same magnetic field range, thus an improvement of the sensitivity of such a device. An equivalent piezomagnetic model aiming at assessing the magnetoelastic coupling in ferromagnetic thin film coated on a piezoelectric substrate was developed and used to predict the velocity shift obtained for a quasi-shear surface acoustic wave propagating on a lithium tantalate $\mathrm{Y}^{\circ} 6^{\circ}$ substrate cut. The velocity shift shape dependent of the magnetic field was perfectly coherent with the experimental measurements. This shape is close to the $c_{66}$ elastic stiffness constant behaviour towards a bias magnetic field, involved in the shear velocity of the magnetostrictive thin film.

\section{ACKNOWLEDGMENT}

The authors would like to thank the support of the Agence Nationale de la Recherche through grant 2010 BLAN 923 0 , RENATECH NETWORK and Centrale Lille through PhD fellowship.

\section{REFERENCES}

[1] N. Tiercelin, A. Talbi, V. Preobrazhensky, P. Pernod, V. Mortet, K. Haenen, and A. Soltani, "Magnetoelectric effect near spin reorientation transition in giant magnetostrictive-aluminum nitride thin film structure," Applied Physics Letters, vol. 93, no. 16, pp. 1-4, 2008.

[2] N. Tiercelin, V. Preobrazhensky, P. Pernod, and A. Ostaschenko, "Enhanced magnetoelectric effect in nanostructured magnetostrictive thin film resonant actuator with field induced spin reorientation transition," Applied Physics Letters, vol. 92, no. 6, p. 62904, 2008.

[3] A. Piorra, R. Jahns, I. Teliban, J. L. Gugat, M. Gerken, R. Knöchel, and E. Quandt, "Magnetoelectric thin film composites with interdigital electrodes," Applied Physics Letters, vol. 103, no. 3, 2013.

[4] O. Bou Matar, N. Gasmi, H. Zhou, M. Goueygou, and A. Talbi, "Legendre and Laguerre polynomial approach for modeling of wave propagation in layered magneto-electro-elastic media," J. Acoust. Soc. Am., vol. 133, no. 3, 2012. 
[5] H. Zhou, A. Talbi, N. Tiercelin, and O. Bou Matar, "Multilayer magnetostrictive structure based surface acoustic wave devices," Applied Physics Letters, vol. 104, no. 11, p. 114101, 2014.

[6] O. Bou Matar, J. F. Robillard, J. O. Vasseur, A.-C. Hladky-Hennion, P. A. Deymier, P. Pernod, and V. Preobrazhensky, "Band gap tunability of magneto-elastic phononic crystal," Journal of Applied Physics, vol. 111, no. 5, p. 54901, 2012. 